\title{
Androgen regulation of the cell-cell adhesion molecule-1 (Ceacam1) gene
}

\author{
Dillon Phan $^{\text {a }}$, Xiaomei Sui ${ }^{\text {, }}$ Dung-Tsa Chen ${ }^{c}$, Sonia M. Najjar ${ }^{\text {d }}$, Guido Jenster ${ }^{\mathrm{e}}$, \\ Sue-Hwa Lin ${ }^{\text {a,* }}$ \\ ${ }^{a}$ Department of Molecular Pathology, The University of Texas, M.D. Anderson Cancer Center, 1515 Holcombe Boulevard, Houston, \\ TX 77030, USA \\ ${ }^{\mathrm{b}}$ Department of Urology, The University of Texas, M.D. Anderson Cancer Center, 1515 Holcombe Boulevard, Houston, TX 77030, USA \\ ${ }^{\mathrm{c}}$ Medical Statistics Section, Division of Hematology/Oncology, Department of Medicine, The University of Alabama at Birmingham, \\ Birmingham, AL, USA \\ d Department of Pharmacology and Therapeutics, Medical College of Ohio, 3035 Arlington Avenue, Toledo, OH, USA \\ e Department of Urology, Erasmus University Rotterdam, PO Box 1738, 3000 DR Rotterdam, The Netherlands
}

Received 25 January 2001; accepted 26 July 2001

\begin{abstract}
Previous studies have established that the cell-cell adhesion molecule-1 (CEACAM1, previously known as C-CAM1) functions as a tumor suppressor in prostate cancer and is involved in the regulation of prostate growth and differentiation. However, the molecular mechanism that modulates CEACAM1 expression in the prostate is not well defined. Since the growth of prostate epithelial cells is androgen-regulated, we investigated the effects of androgen and the androgen receptor (AR) on CEACAM1 expression. Transient transfection experiments showed that the AR can enhance the Ceacam1 promoter activity in a ligand-dependent manner and that the regulatory element resides within a relatively short ( -249 to $-194 \mathrm{bp}$ ) segment of the $5^{\prime}$-flanking region of the Ceacam 1 gene. This androgen regulation is likely through direct AR-promoter binding because a mutant AR defective in DNA binding failed to upregulate reporter gene expression. Furthermore, electrophoretic mobility shift assays demonstrated that the AR specifically binds to this sequence, and mutation analysis of the potential ARE sequences revealed a region within the sequence that was required for the AR to activate the Ceacam1 gene. Therefore, the regulation of Ceacam 1 gene expression by androgen may be one of the mechanisms by which androgen regulates prostatic function. (C) 2001 Elsevier Science Ireland Ltd. All rights reserved.
\end{abstract}

Keywords: CEACAM1; Cell adhesion molecule; Androgen receptor; Tumor suppressor; Prostate

\section{Introduction}

The cell-cell adhesion molecule-1 (C-CAM1), recently renamed CEACAM1 (Beauchemin et al., 1999), is a member of the immunoglobulin supergene family (Lin and Guidotti, 1989; Lin et al., 1991). CEACAM1 is mainly expressed in epithelial cells of many different tissues, including the prostate (Odin et al., 1988). Loss of CEACAM1 expression is an early event in prostate cancer progression (Kleinerman et al., 1995; Pu et al.,

\footnotetext{
* Corresponding author. Tel.: + 1-713-794-1559; fax: + 1-713-7944672 .

E-mail address: slin@notes.mdacc.tmc.edu (S.-H. Lin).
}

1999), suggesting that this molecule may play an important role in prostate tumorigenesis. Consistent with this hypothesis, expression of CEACAM1 in prostate cancer cells can suppress their tumorigenicity in vivo (Estrera et al., 1999; Hsieh et al., 1995; Luo et al., 1999). These observations suggest that CEACAM1 functions as a tumor suppressor in prostate cancer.

The prostate is an androgen-dependent organ, as androgen is the major regulator of prostate development, growth, and secretory function. Induction of prostate involution using androgen ablation is one of the most effective treatments of late-stage prostate cancer. Since CEACAM1 is a tumor suppressor in prostate cancer, it is important to know whether expression of CEACAM1 in the prostate is regulated by androgen. 
The rat Ceacam 1 promoter belongs to the GC-rich class of TATA-less promoters (Najjar et al., 1996). Deletion and substitution analyses have revealed that the three proximal Sp1 binding sites are essential for basal transcription of the Ceacam 1 gene. In addition, Najjar et al. (1996) have shown that Ceacam1 promoter activity is stimulated 2-3-fold by insulin, dexamethasone, and cyclic adenosine monophosphate treatment. However, the effect of androgen on Ceacam 1 promoter activity has not been examined. Therefore, in this study, we examined whether the androgen receptor (AR) regulates Ceacam 1 promoter activity.

\section{Materials and methods}

\subsection{Plasmid constructions}

The 5'-flanking region of the rat Ceacam 1 gene was cloned as described previously (Najjar et al., 1996). Nucleotides were numbered relative to +1 at the ATG translation initiation codon and labeled as negative numbers to reflect their position upstream $\left(5^{\prime}\right)$ of the ATG site. Using polymerase chain reaction (PCR), 5' deletion products $(-1609,-439,-249,-194$, $-147,-131,-124$ and -112 bp) of the Ceacam 1 gene were synthesized and subcloned at the XhoI and HindIII sites of the pGL3-BASIC plasmid (Promega, Madison, WI) (Najjar et al., 1996).

The mutants -249 pLucARE-1Mut and -249 pLucARE-2Mut were generated by site-directed mutagenesis of the -249 pLuc vector using PCR. Oligo \# 305 (reverse primer; AAGCTTTTCTCTTGGGGAAGA) and oligo \# 306 (forward primer; CTCGAGATGTTCTAGAACAATGAACCGAAAAGAGATCCCGCGAAGGATGGGAGGACAGCA) were used as primers to introduce substitutions into the ARE-1 region, while oligo \# 307 (forward primer; GCTAGCCCGGGCTCGAGAGTCGACAGAACAATGAACCGAAAA) and oligo \#305 were used to introduce substitutions into the ARE-2 region; the sequences that were changed from the wild type are underlined. After these PCR products were sequenced to confirm the mutations, they were subcloned at the XhoI and HindIII sites of the pGL3-BASIC plasmid. The construction of the reporter plasmid harboring two androgen response elements and a TATA box driving the luciferase gene (p[ARE]2-E1bluc) has been described previously (Jenster et al., 1997).

The human AR cDNA expression vector $\left(\mathrm{pAR}_{0}\right)$ was constructed using the simian virus 40 (SV40) early promoter and the rabbit $\beta$-globin polyadenylation signal as described previously (Brinkmann et al., 1989). The AR mutant expression vector pAR64, in which the first zinc finger in the AR was disrupted by the replacement of two cysteines with serine and phenylalanine, was constructed as described by Jenster et al. (1993).
Additionally, the superactive AR expression vector pcDNA-AR ${ }_{0}$ p65 was constructed by inserting the Asp 718-(filled in with the Klenow fragment) and Sac II digested fragment of pcDNA-AR $\mathrm{LBD}_{\mathrm{L}}-\mathrm{p} 65$ into the $\mathrm{HpaI}$ and $\mathrm{Sac}$ II digested pcDNA-AR $\mathrm{mcs}$ vector (Sui et al., 1999). This resulted in the generation of a fusion protein containing the wild-type AR fused with the transactivation domain of $\mathrm{p} 65 / \mathrm{RelA}$.

\subsection{Culture and transfection of HeLa cells}

HeLa human cervical carcinoma cells (American Type Culture Collection, Manassas, VA) were maintained in minimal essential medium supplemented with $10 \%(\mathrm{v} / \mathrm{v})$ fetal calf serum (FCS). These cells $(50000)$ were plated in a 12-well plate with $10 \%(\mathrm{v} / \mathrm{v})$ charcoalstripped FCS $24 \mathrm{~h}$ before transfection. The cells were transfected with $0.3 \mu \mathrm{g}$ of both luciferase reporter plasmid containing a Ceacam 1 promoter fragment and a receptor plasmid containing either wild-type $\left(\mathrm{pAR}_{0}\right)$ or modified AR (pAR64 or pcDNA-AR ${ }_{0}$ p65) per well using Lipofectin (Life Technologies, Inc., Grand Island, NY) according to the manufacturer's guidelines. About $24 \mathrm{~h}$ after transfection, the cells were washed and fed with medium containing charcoal stripped serum with or without R1881 (17 $\alpha$-methyltrienolone; NEN Life Science Products, Boston, MA), and the incubations were continued for an additional $24 \mathrm{~h}$. The cells were then lysed in $200 \mu \mathrm{l}$ lysis buffer, and the luciferase activity was measured using a luciferase assay system (Promega). The experiments were performed in triplicate.

\subsection{Electrophoretic mobility shift assay (EMSA)}

EMSA was carried out using a bandshift assay system (Promega). Oligonucleotides having sequences corresponding to the region between -194 to $-249 \mathrm{bp}$ of the Ceacam 1 promoter were synthesized by Genosys (Houston, TX) and used as probe. In addition, oligonucleotides containing the AR consensus sequence were purchased from Santa Cruz Biotechnology (Santa Cruz, CA) and used as competitors. The plasmid pRSETGST-AR $\mathrm{DBD}_{\text {DB }}$ containing a sequence from the AR DNA binding domain fused to GST was constructed by inserting $0.3 \mathrm{~kb}$ of the Klenow-treated $R s r \mathrm{II} / X b a \mathrm{I}$ digested ARDBD fragment from AR126 (Jenster et al., 1995) into the Klenow-treated NcoI/HindIII digested pRSET-GST-SRC782-1139 vector (Spencer et al., 1997). The GST-fusion protein containing the AR

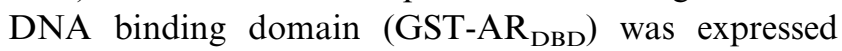
and purified from Escherichia coli BL21( $\lambda$ DE3), and $100 \mathrm{ng}$ of GST-AR ${ }_{\text {DBD }}$ protein was used for EMSA. Purified GST protein was used as a negative control. 


\subsection{Statistic analysis}

Student's $t$-test was used to examine R1881 effects on different types of mutation. We chose the ratio of promoter activities in the presence and absence of R1881 as a dependent variable to avoid variation in basal activity between different experiments.

\section{Results}

\subsection{Localization of an androgen-responsive region in the Ceacam 1 promoter}

To examine the effect of AR on Ceacam 1 promoter activity, we first tested cell lines that express AR. Although LNCaP cells, which were isolated from the lymph node metastasis of a prostate cancer patient (Horoszewicz et al., 1983), were shown to express AR, the transfection efficiency in this cell line was very low (data not shown). Another prostatic cell line that express $\mathrm{AR}$ is $\mathrm{NbE}$ cell. $\mathrm{NbE}$ cell is a cell line derived from the ventral prostate of Noble rat and is shown to express AR (Chung et al., 1989). We found that the reporter plasmid containing two androgen response elements (p[ARE $]_{2}$-E1b-luc) could not respond to R1881 stimulation when transfected into the NbE cells (data not shown). However, this reporter was activated 90-340-folds by R1881 when it was co-transfected with a wild type AR plasmid in the NbE cells (data not shown). This observation suggested that the AR in $\mathrm{NbE}$ cells was not functional. The reason for $\mathrm{AR}$ dysfunction in NbE cells is not known. Previous studies by Jenster et al. (1995) and Sui et al. (1999) have shown that HeLa cells co-transfected with AR and promoter constructs were suitable for AR related studies. As a result, we chose to use HeLa cells co-transfected with AR for this study.

Ceacam 1 promoters with different lengths that were constructed by $5^{\prime}$ deletion were cloned in front of the luciferase gene in the reporter plasmid. Each of these plasmids was transiently cotransfected with the AR expression vector $\mathrm{pAR}_{0}$ into HeLa cells; the reporter plasmid containing two androgen response elements and a TATA box derived from the E1b gene (p[ARE]2E1b-luc) was used as a positive control. In the absence of the androgen analogue R1881, the 1609 bp Ceacam 1 promoter mediated a 106-fold increase in reporter gene

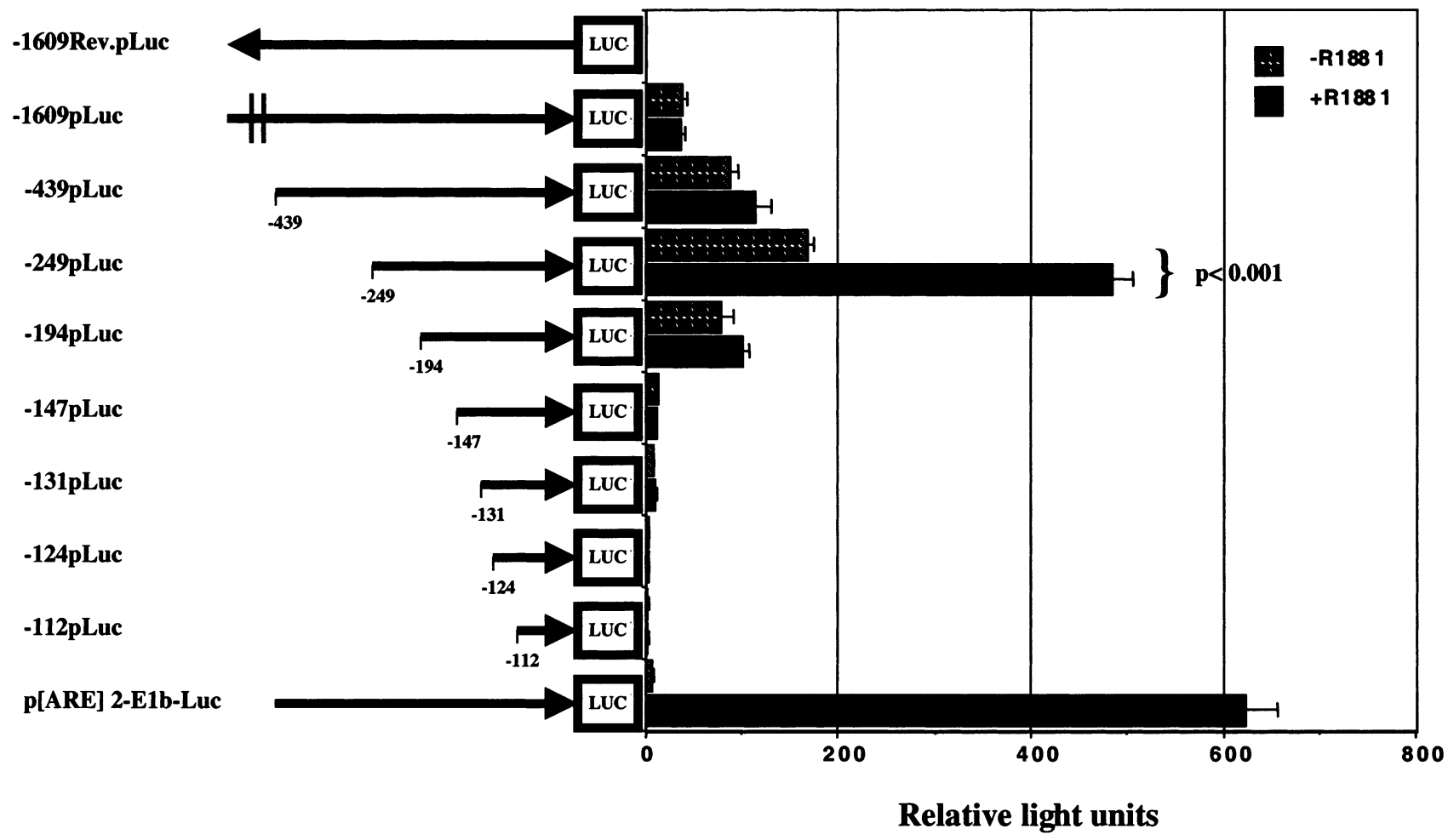

Fig. 1. Regulation of CEACAM1 expression by androgen. A series of reporter plasmids containing Ceacam1 promoter fragments having different $5^{\prime}$ deletions were cotransfected with a wild-type AR plasmid $\left(\mathrm{pAR}_{0}\right)$ into HeLa cells. About $24 \mathrm{~h}$ after transfection, the cells were incubated with $(+)$ or without $(-) 1 \mathrm{nM} \mathrm{R} 1881$. The luciferase activity of these cell lysates was determined as described in Section 2 . This experiment was repeated eight times with triplicate transfections for each construct and similar results were obtained. Results from one of these experiments were shown and the luciferase activities were reported as the average \pm S.D. in relative light units of triplicate transfections. Statistic analysis was used to determine whether there was difference between the R1881 treated and untreated groups. Only $-249 \mathrm{pLuc}$ construct showed statistically significant difference and the $P$ value for -249 pLuc is shown. 


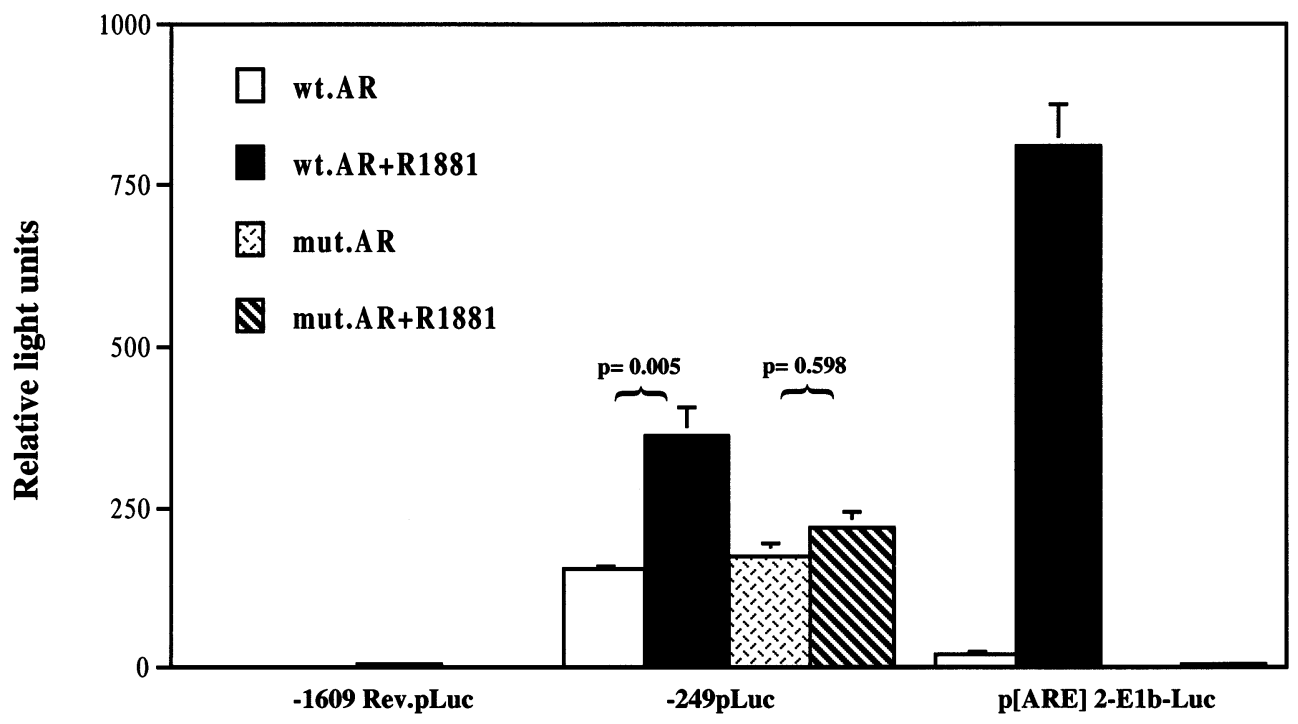

Fig. 2. Effect of an AR mutation on its ability to activate the Ceacam1 promoter. Cells were transfected with the -249 pLuc Ceacam 1 promoter together with a wild-type $\mathrm{AR}\left(\mathrm{pAR}_{0}\right)$ or mutant $\mathrm{AR}$ (pAR64) plasmid, respectively. The data are presented as the mean \pm S.E. of three independent experiments. Statistic analysis was used to determine whether there was difference between the R1881 treated and untreated groups. The $P$ values for each group are shown.

expression as compared with the reverse-oriented Ceacam 1 promoter fragment (Fig. 1). Deletion of the region between nt -1609 and -439 induced a slight increase in the basal promoter activity (Fig. 1), suggesting that this region may contain potential down-regulators. Deletion of the Ceacam 1 promoter up to -194 bp did not abolish its ability to induce luciferase expression, while deletion up to -147 bp markedly reduced its promoter activity. This result suggested that a minimal promoter is located within the first $194 \mathrm{bp} 5^{\prime}$ from Ceacam1's translation start site. We next investigated whether androgen had an effect on the Ceacam 1 promoter. As shown in Fig. 1, the plasmid containing the Ceacam 1 promoter region from -249 to $-21 \mathrm{bp}$ exhibited a 2.5 -fold increase in luciferase activity upon the addition of the androgen analogue R1881. In contrast, no significant hormone response was observed with plasmids containing the entire 1609,439 , or $194 \mathrm{bp}$ segment proximal to the translation start site. These observations suggested that the region from -249 to - 194 bp in the Ceacam 1 gene may contain an androgen-regulated sequence.

\subsection{Direct binding of the AR to the promoter sequence}

The AR is a $110-112 \mathrm{kDa}$ protein containing transcriptional activation domains in its $\mathrm{N}$-terminal region, a centrally located DNA binding domain, and the ligand binding domain at its C-terminus (Jenster et al., 1991). To test whether activation of the Ceacam 1 promoter by androgen is due to direct interaction between it and the AR, we investigated the effect of a mutant AR, AR64, which is defective in DNA binding (Jenster et al., 1993), on Ceacam1 promoter activity. In contrast to the wild-type AR, AR64, when cotransfected with -249 pLuc into HeLa cells, did not show significant hormone induction (Fig. 2). Similarly, p[ARE]2-E1bLuc lost its response to R1881 stimulation. These results suggest that activation of the Ceacam 1 promoter by the wild-type AR requires its DNA binding domain; thus, AR may bind directly to Ceacam 1 promoter.

In addition, EMSA was used to determine whether the AR can bind to the promoter sequence. A doublestranded oligonucleotide containing the promoter sequence from -249 to $-194 \mathrm{bp}$ was used in the assay. Fig. 3 shows that the AR DNA binding domain can bind to the oligonucleotide ( -249 to $-194 \mathrm{bp}$ ) and that the binding can be specifically competed by the unlabeled corresponding oligonucleotide duplexes, as well as an unlabeled oligonucleotide containing the AR consensus sequence (Roche et al., 1992). This observation suggested that the AR binds specifically to the Ceacam 1 promoter sequence.

\subsection{Identification of AR-interacting sites}

Using a DNA binding site-selection assay, Roche et al. (1992) determined a consensus AR DNA binding site for the AR. Two regions in the Ceacam 1 promoter, located at -215 to $-220 \mathrm{bp}$ and -243 to $-248 \mathrm{bp}$, respectively, showed homology to the consensus halfsite sequence and could be responsible for androgen induction of the -249 pLuc reporter activity (Fig. 4). These two potential AR binding sites (ARE-1 and ARE-2) were mutated to see if they are indeed involved in androgen regulation. The effect of mutating ARE-1 
or ARE-2 on the promoter activity was examined. Mutations of ARE-1 did not cause a significant change in the Ceacam 1 promoter's response to R1881, while mutation of ARE-2 completely abolished the response (Fig. 4). In addition, mutating both ARE-1 and ARE-2 had a similar effect to that of mutating ARE-2 alone. These observations suggested that only ARE-2 is involved in the androgen regulation of Ceacam1 promoter activity.

A mutational analysis of potential ARE sites was also performed using a superactive AR containing the AR fused with the transactivation domain of p65/RelA (Schmitz and Baeuerle, 1991). As part of the $A_{0} \mathrm{p}_{0} 65$ fusion protein, the p65 activation domain can recruit additional coactivators and proteins of the preinitiation complex resulting in amplification of AR-mediated transcriptional signals. As shown in Fig. 5A, the -249 bp Ceacam 1 promoter activity showed a 5-6-fold increase in response to R1881 stimulation with the superactive AR in contrast to a $2-3$-fold increase in response

\section{C-CAM1 (-249 to-194 bp) Probe}

$\begin{array}{rlllll}\text { Lane } & 1 & 2 & 3 & 4 & 5 \\ \text { GST } & - & + & - & - & - \\ \text { GST-AR } & - & - & + & + & + \\ 50 \times(-249 \text { to }-194 \text { bp) competitor } & - & - & - & + & - \\ 50 x \text { AR consensus competitor } & - & - & - & - & +\end{array}$

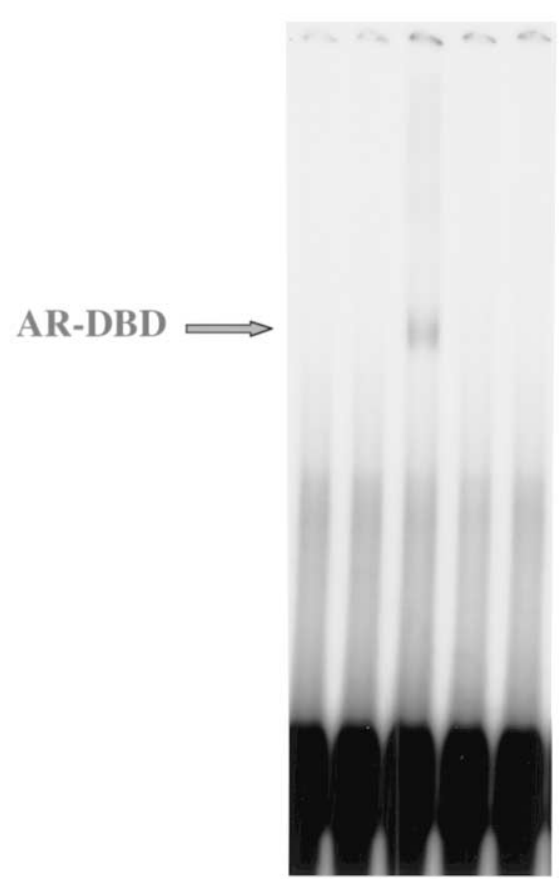

Fig. 3. EMSA, which was carried out using purified GST-AR ${ }_{\mathrm{DBD}}$ and the labeled double-stranded oligonucleotide probe containing a sequence from -249 to $-194 \mathrm{bp}$ of the Ceacam 1 promoter. Lane 1, without protein; lane 2, with GST protein; lane 3, with GST-AR ${ }_{\mathrm{DBD}}$; lane 4, with GST-AR ${ }_{\text {DBD }}$ and a 50-fold molar excess of the unlabeled probe; lane 5, with GST-AR ${ }_{\mathrm{DBD}}$ and a 50-fold molar excess of a double-stranded oligonucleotide containing the AR consensus sequence (Roche et al., 1992). to R1881 stimulation with the wild-type AR. Such an enhancement of reporter activity was used to further confirm the mutational analysis. In the presence of the superactive AR, mutation of ARE-1 resulted in a 4-fold increase in luciferase activity in response to R1881. As observed with wild-type AR, R1881 treatment did not increase the promoter activity of the ARE-2 mutant or combined ARE-1/ARE-2 mutant. These observations further confirmed that the AR only requires ARE-2 to stimulate Ceacam 1 promoter activity.

\section{Discussion}

Androgen is the most important factor that regulates prostate growth and differentiation. A series of genes that have functions related to cell-growth modulation have been shown to be regulated by androgen in prostate cells. It was shown that androgen can directly or indirectly upregulate growth factors such as epidermal growth factor (Hiramatsu et al., 1988; Nishi et al., 1996), keratinocyte growth factor (Fasciana et al., 1996; Peehl and Rubin, 1995; Rubin et al., 1995; Yan et al., 1992), and basic fibroblast growth factor (Katz et al., 1989; Zuck et al., 1992), leading to epithelial-cell proliferation. In addition, transforming growth factor $\beta$, which has been linked to programmed cell death, is induced upon androgen withdrawal (Kyprianou et al., 1990). Regulation of growth hormones and apoptotic factors may contribute to the growth of the prostate. On the other hand, androgen upregulation of insulinlike growth factor binding proteins (IGFBP) could make the potent prostate mitogens IGF-I and IGF-II unavailable for growth induction (Gregory et al., 1999). Cell-cycle regulatory proteins such as cdk2, cdk4, cyclin D3, cyclin A, p21CIP1/WAF-1, p27kip1, and p16 were also found to be regulated by androgen (Gregory et al., 1998; Knudsen et al., 1998; Kokontis et al., 1998; Lu et al., 1999, 1997). These diverse androgen-regulated events result in the maintenance of prostate homeostasis; disruption of these intricately balanced events may lead to prostate cancer initiation and progression.

In the present study, we showed that Ceacam1, a tumor suppressor gene, can, under defined circumstances and/or in a specific cellular context, be regulated by androgen. Specifically, androgen could up-regulate CEACAM1 expression in a ligand-dependent manner when tested in vitro. This androgen regulation is controlled by only one of the two half-sites of the AR consensus sequence (Roche et al., 1992). A similar event was also observed by Dai and Burnstein (1996), who showed that the presence of one half-site of the AR consensus sequence is sufficient to upregulate the promoter of the AR gene by the AR. This half-site interaction may not provide as strong an activity as that provided by the full consensus sequence in the 


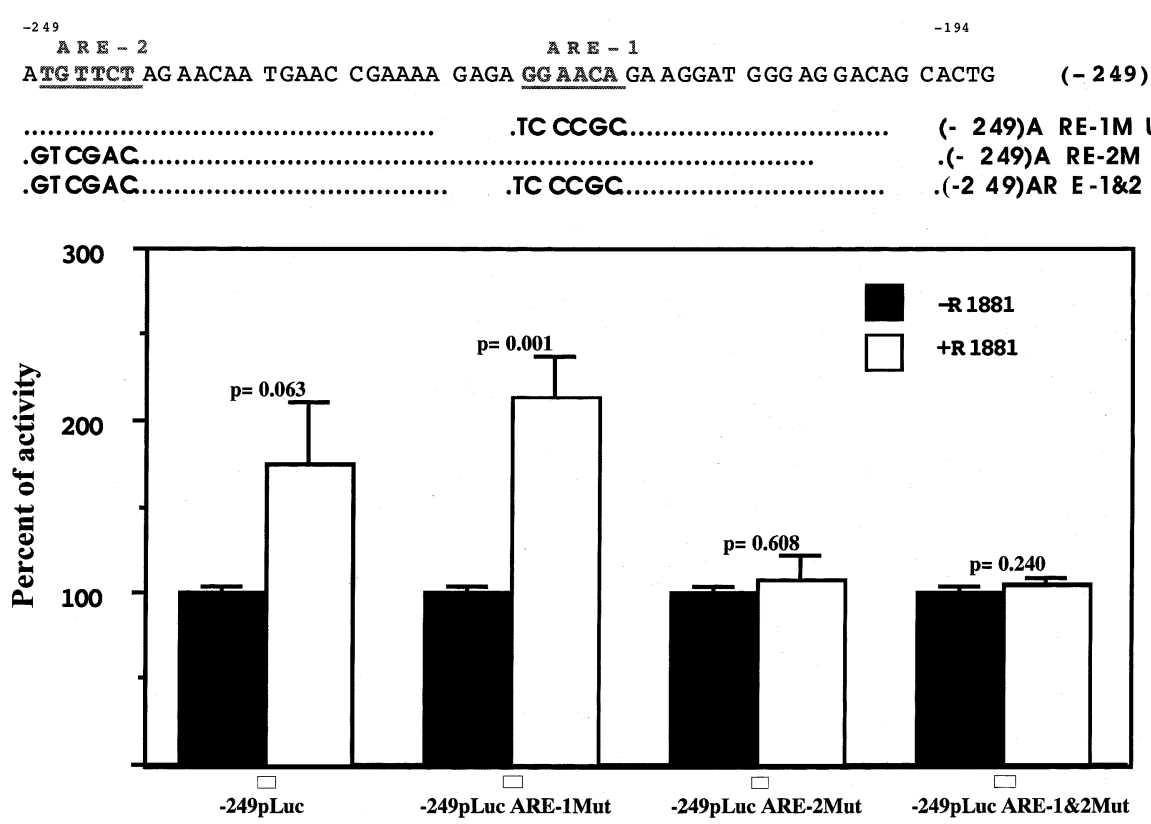

Fig. 4. Mutation analysis of the putative AREs in the Ceacam1 promoter. The nucleotide sequence from -249 to -194 bp in the rat Ceacam 1 promoter is shown. Two half-sites of putative androgen-responsive elements (ARE-1 and ARE-2) are underlined; the mutated sequence of the two elements is shown. Cells were transfected with reporter plasmids containing the -249 bp promoters, whose putative ARE-1 or ARE-2 sequence was mutated as described in Section 2. The luciferase activity is presented as a percent of that of the corresponding plasmid containing no mutation and without R1881 treatment. The data are presented as the mean \pm S.E. of three independent experiments. Statistic analysis was used to determine whether there was difference between the R1881 treated and untreated groups. The $P$ values for each group are shown.

probasin promoter (Kasper et al., 1994) and PSA promoter (Zhang et al., 1997). In addition, the longer Ceacam 1 promoter, i.e. the 439-bp segment, did not show ligand-dependent regulation by androgen, suggesting that other regulatory mechanisms may be present upstream of the promoter region and may influence the interaction of the AR with the Ceacam 1 proximal promoter region. Thus, the regulation of CEACAM1 expression by the AR is complex and may depend on its cellular context. In this regard, it is interesting to note that in the rat, CEACAM1 is mainly expressed in the dorsal lobe but not the ventral lobe of the prostate, although the AR is expressed in both prostate lobes (Makarovskiy et al., 1999). In addition, using castration-induced prostate involution followed by administration of androgen, we have previously shown that expression of CEACAM1 in the rat ventral prostate was negatively regulated by androgen in vivo, while its expression in the dorsal prostate showed no response to androgen manipulation in vivo (Hsieh and Lin, 1994; Makarovskiy et al., 1999). In the mouse prostate, on the other hand, increased CEACAM1 expression was detected in the dorsal prostate lobe following castration (Pu et al., 1999). In the present study, we found that androgen could up-regulated CEACAM1 expression in a ligand-dependent manner, although up-regulation of CEACAM1 expression by androgen was observed only with a relatively short promoter (bp -294 to -197$)$. Together, these obser- vations suggest that the regulation of CEACAM1 expression by androgen in vivo is a complex process. Although the AR has a positive effect on the proximal region of the Ceacam 1 promoter, it may have a negative effect on the other region of the Ceacam 1 promoter. AR regulation of CEACAM1 expression is, therefore, influenced by the cellular context and possibly the stromal components of the prostate. The interactions between prostatic epithelial and stromal components have been shown to contribute to androgen-dependent regulation of prostate growth and differentiation (Chung, 1995; Cunha et al., 1987). As a result, although direct interactions between AR and Ceacam 1 promoter may contribute to androgen regulation of CEACAM1 expression, other factors including signals from stromal-epithelial interactions may also influence CEACAM1 expression in vivo.

Primary prostate cancers are largely dependent on androgens for growth and survival. Therefore, the disease responds favorably to androgen ablation and antiandrogen therapy in most patients. However, virtually all patients will relapse with clinically defined androgenindependent cancer. Previously, the role of CEACAM1 in androgen-dependent and androgen-independent prostate cancer was studied by examining the patterns of CEACAM1 expression during prostate cancer initiation, progression, and metastasis in the transgenic adenocarcinoma of mouse prostate (TRAMP) model, which was generated by using the rat probasin pro- 
moter to target the SV40 large T antigen specifically to the mouse prostate (Greenberg et al., 1995). In the TRAMP mice, immunohistochemical staining using polyclonal antibody Ab669 against CEACAM1 revealed that the CEACAM1 protein was expressed in normal prostate epithelia, as well as low-grade prostate intraepithelial neoplasia (PIN); the expression was uniform on the luminal surfaces of these epithelia. CEACAM1 expression was noticeably reduced and the staining pattern was heterogeneous in some cases of high-grade PIN, and CEACAM1 staining was generally absent from prostate cancer and metastatic lymph nodes. Androgen-independent prostate cancer and its metastases generated in castrated TRAMP mice were also CEACAM1 negative (Pu et al., 1999). Since loss of CEACAM1 expression occurred before the development of androgen-independent tumors, it is likely that the AR regulation of CEACAM1 expression is not related to the loss of CEACAM1 during prostate cancer progression.

Other factors that have been shown to have an effect on the Ceacam 1 promoter include the upstream stimu-

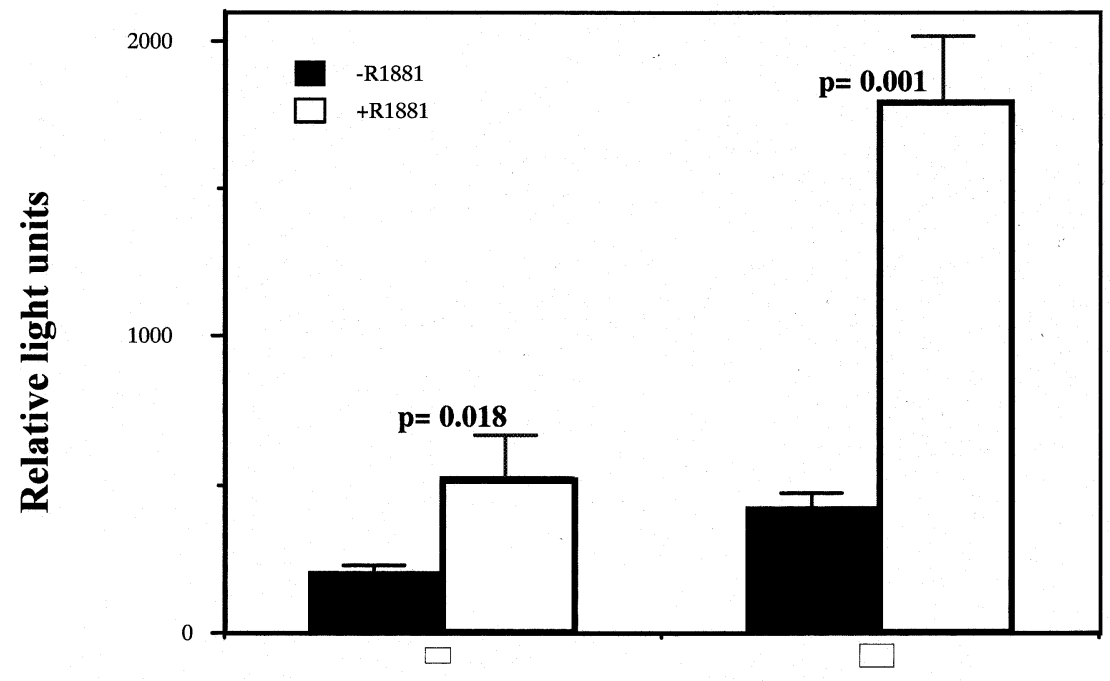

(A)

AR

Superactive AR

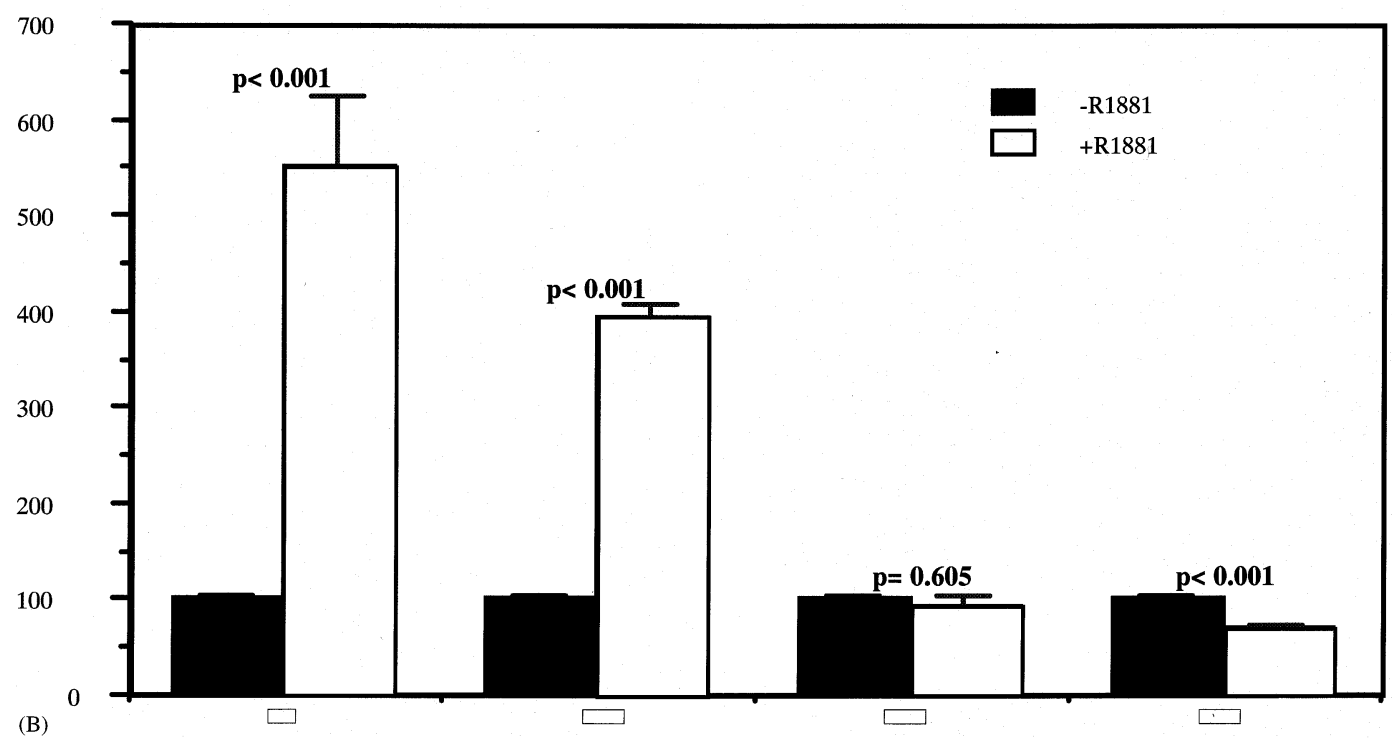

Fig. 5. Activation of Ceacam 1 promoter activity by a superactive $A R\left(\mathrm{AR}_{0} \mathrm{p} 65\right)$. (A) The Ceacam1 promoter transcription activity was examined using cotransfection of the Ceacam 1 promoter reporter construct $(-249 \mathrm{pLuc})$ and the wild-type or superactive $A R$ expression plasmid (pAR $\left.\mathrm{p}_{05}\right)$ into HeLa cells. The luciferase activity was determined from cell lysates of transfected cells as described in Section 2 . This experiment was repeated six times with triplicate transfections for each construct and similar results were obtained. Results from one of these experiments were shown and the luciferase activities were reported as the average \pm S.D. in relative light units of triplicate transfections. (B) Effect of superactive AR on mutant Ceacam 1 promoter transcription activity. The luciferase activity is presented as a percent of the luciferase activity without R1881 treatment. The data are presented as the mean \pm S.E. of three independent experiments. Statistic analysis were performed as described in Section 2 to compare the R1881-treated and untreated groups for each construct, and the $P$ values for each group are shown. 
latory factor and hepatocyte nuclear factor-4 (Hauck et al., 1994). Also, Chen et al. (1996) showed that treatment of HT-29 cells with interferon- $\gamma$ (IFN- $\gamma$ ) upregulated CEACAM1 expression. This was due to the ability of IFN- $\gamma$ to upregulate the expression of IRF-1, which, by binding to the interferon stimulated response element located in the human Ceacam 1 promoter, activated Ceacam 1 transcription. Thus, the regulation of CEACAM1 expression is a combination of different transcriptional factors, one of which is the AR.

\section{Acknowledgements}

This work was supported by grants CA64856 from the National Institutes of Health and DAMD17-98-18465-1 from the U.S. Department of the Army and predoctoral training grant T32 CA67759 from the National Institutes of Health to Dillon Phan The nucleotide sequences used in this paper are in the GenBank/EMBL Data Bank with accession number U27207 for the $5^{\prime}$-flanking region of the rat CEACAM1/pp120 gene.

\section{References}

Beauchemin, N., Draber, P., Dveksler, G., Gold, P., Gray-Owen, S., Grunert, F., Hammarstrom, S., Holmes, K.V., Karlsson, A., Kuroki, M., Lin, S.-H., Lucka, L., Najjar, S.M., Neumaier, M., Obrink, B., Shively, J.E., Skubitz, K.M., Stanners, C.P., Thomas, P., Thompson, J.A., Virji, M., von Kleist, S., Wagener, C., Watts, S., Zimmermann, W., 1999. Redefined nomenclature for members of the carcinoembryonic antigen family. Exp. Cell Res. 252, 243-249.

Brinkmann, A.O., Faber, P.W., van Rooij, H.C.J., Kuiper, G.G.J.M., Ris, C., Klaassen, P., van der Korput, J.A.G.M., Voorhorst, M.M., van Laar, J.H., Mulder, E., Trapman, J., 1989. The human androgen receptor: domain structure, genomic organization and regulation of expression. J. Steroid Biochem. 34, 307-310.

Chen, C.-J., Lin, T.-T., Shively, J.E., 1996. Role of interferon regulatory factor-1 in the induction of biliary glycoprotein (Cell CAM1) by interferon-r. J. Biol. Chem. 271, 28181-28188.

Chung, L.W.K., 1995. The role of stromal-epithelial interaction in normal and malignant growth. Cancer Surv. 23, 33-42.

Chung, L.W.K., Chang, S.-M., Bell, C., Zhau, H.E., Ro, J.Y., von Eschenbach, A.C., 1989. Co-inoculation of tumorigenic rat prostate mesenchymal cells with non-tumorigenic epithelial cells results in the development of carcinosarcoma in syngeneic and athymic animals. Int. J. Cancer 43, 1179-1187.

Cunha, G.R., Donjacour, A.A., Cooke, P.S., Mee, S., Bigsby, R.M., Higgins, S.J., Sugimura, Y., 1987. The endocrinology and developmental biology of the prostate. Endocr. Rev. 8, 338-362.

Dai, J.L., Burnstein, K.L., 1996. Two androgen response elements in the androgen receptor coding region are required for cell-specific up-regulation of receptor messenger RNA. Mol. Endocrinol. 10, $1582-1594$.

Estrera, V.T., Luo, W., Phan, D., Hixson, D., Lin, S.-H., 1999. The cytoplasmic domain of C- CAM1 is necessary and sufficient in suppression the growth of prostate cancer cells. Biochem. Biophys. Res. Commun. 263, 797-803.
Fasciana, C., van der Made, A.C., Faber, P.W., Trapman, J., 1996. Androgen regulation of the rat keratinocyte growth factor (KGF/ FGF7) promoter. Biochem. Biophys. Res. Commun. 220, 858863.

Greenberg, N.M., DeMayo, F., Finegold, M.J., Medina, D., Tilley, W.D., Aspinall, J.O., Cunha, G.R., Donjacour, A.A., Matusik, R.J., Rosen, J.M., 1995. Prostate cancer in a transgenic mouse. Proc. Natl. Acad. Sci. USA 92, 3439-3443.

Gregory, C.W., Hamil, K.G., Kim, D., Hall, S.H., Pretlow, T.G., Mohler, J.L., French, F.S., 1998. Androgen receptor expression in androgen-independent prostate cancer is associated with increased expression of androgen-regulated genes. Cancer Res. 58, 57185724.

Gregory, C.W., Kim, D., Ye, P., D'Ercole, A.J., Pretlow, T.G., Mohler, J.L., French, F.S., 1999. Androgen receptor up-regulates insulin-like growth factor binding protein-5 (IGFBP-5) expression in a human prostate cancer xenograft. Endocrinology 140, 2372 2381.

Hauck, W., Nedellec, P., Turbide, C., Stanners, C.P., Barnett, T.R., Beauchemin, N., 1994. Transcriptional control of the human biliary glycoprotein gene, a CEA gene family member down-regulated in colorectal carcinomas. Eur. J. Biochem. 223, 529-541.

Hiramatsu, M., Kashinmata, M., Minami, N., Sato, A., Murayama, M., 1988. Androgenic regulation of epidermal growth factor in the mouse ventral prostate. Biochem. Int. 2, 311-317.

Horoszewicz, J.S., Leong, S.S., Kawinski, E., Karr, J.P., Rosenthal, H., Chu, T.M., Mirand, E.A., Murphy, G.P., 1983. LNCaP model of human prostatic carcinoma. Cancer Res. 43, 1809-1818.

Hsieh, J.T., Lin, S.H., 1994. Androgen regulation of cell adhesion molecule gene expression in rat prostate during organ degeneration. C-CAM belongs to a class of androgen-repressed genes associated with enriched stem/amplifying cell population after prolonged castration. J. Biol. Chem. 269, 3711-3716.

Hsieh, J.T., Luo, W., Song, W., Wang, Y., Kleinerman, D.I., Van, N.T., Lin, S.H., 1995. Tumor suppressive role of an androgenregulated epithelial cell adhesion molecule (C-CAM) in prostate carcinoma cell revealed by sense and antisense approaches. Cancer Res. 55, 190-197.

Jenster, G., van der Korput, H.A., van Vroonhoven, C., van der Kwast, T.H., Trapman, J., Brinkmann, A.O., 1991. Domains of the human androgen receptor involved in steroid binding, transcriptional activation, and subcellular localization. Mol. Endocrinol. 5, 1396-1404.

Jenster, G., Trapman, J., Brinkmann, A.O., 1993. Nuclear import of the human androgen receptor. Biochem. J. 293, 761-768.

Jenster, G., van der Korput, H.A.G.M., Trapman, J., Brinkmann, A.O., 1995. Identification of two transcription activation units in the N-terminal domain of the human androgen receptor. J. Biol. Chem. 270, 7341-7346.

Jenster, G., Spencer, T., Burcin, M., Tsai, S.Y., Tsai, M.-J., O'Malley, B.W., 1997. Steroid receptor induction of gene transcription: a two-step model. Proc. Natl. Acad. Sci. USA 94, 7879-7884.

Kasper, S., Rennie, P.S., Bruchovsky, N., Sheppard, P.C., Cheng, H., Lin, L., Shiu, R.P.C., Snoek, R., Matusik, R.J., 1994. Cooperative binding of androgen receptors to two DNA sequences is required for androgen induction of the probasin gene. J. Biol. Chem. 269, 31763-31769.

Katz, A.E., Benson, M.C., Wise, G.J., Olsson, C.A., Bandyk, M.G., Sawczuk, I.S., 1989. Gene activation during the early phase of androgen-stimulated rat prostate regrowth. Cancer Res. 49, 5889-5894.

Kleinerman, D.I., Troncoso, P., Lin, S.H., Pisters, L.L., Sherwood, E.R., Brooks, T., von Eschenbach, A.C., Hsieh, J.T., 1995. Consistent expression of an epithelial cell adhesion molecule (C-CAM) during human prostate development and loss of expression in prostate cancer: implication as a tumor suppressor. Cancer Res. $55,1215-1220$. 
Knudsen, K.E., Arden, K.C., Cavenee, W.K., 1998. Multiple G1 regulatory elements control the androgen-dependent proliferation of prostatic carcinoma cells. J. Biol. Chem. 273, 20213-20222.

Kokontis, J.M., Hay, N., Liao, S., 1998. Progression of LNCaP prostate tumor cells during androgen deprivation: hormone-independent growth, repression of proliferation by androgen, and role for p27kip1 in androgen-induced cell cycle arrest. Mol. Endocrinol. $12,941-953$.

Kyprianou, N., English, H.F., Isaacs, J.T., 1990. Programmed cell death during regression of PC-82 human prostate cancer following androgen ablation. Cancer Res. 50, 3748-3753.

Lin, S.-H., Guidotti, G., 1989. Cloning and expression of a cDNA coding for a rat liver plasma membrane ecto-ATPase: the primary structure of the ecto-ATPase is similar to that of the human biliary glycoprotein. J. Biol. Chem. 264, 14408-14414.

Lin, S.H., Culic, O., Flanagan, D., Hixson, D.C., 1991. Immunochemical characterization of two isoforms of rat liver ecto-ATPase that show an immunological and structural identity with a glycoprotein cell-adhesion molecule with Mr 105 000. Biochem. J. 278, 155-161.

Lu, S., Tsai, S.Y., Tsai, M.-J., 1997. Regulation of androgen-dependent prostatic cancer cell growth: androgen regulation of CDK2, CDK4, and CK1p16 genes. Cancer Res. 57, 4511-4516.

Lu, S., Liu, M., Epner, D.E., Tsai, S.Y., Tsai, M.-J., 1999. Androgen regulation of the cyclin- dependent kinase inhibitor p21 gene through an androgen response element in the proximal promoter. Mol. Endocrinol. 13, 376-384.

Luo, W., Talposky, M., Earley, K., Wood, C., Wilson, D., Logothetis, C.J., Lin, S.-H., 1999. The tumor suppressive activity of CD66a in prostate cancer. Cancer Gene Ther. 6, 313-321.

Makarovskiy, A.N., Pu, Y.-S., Lo, P., Earley, K., Paglia, M., Hixson, D.C., Lin, S.-H., 1999. Expression and androgen regulation of C-CAM cell adhesion molecule isoforms in rat dorsal and ventral prostate. Oncogene 18, 3252-3260.

Najjar, S.M., Boisclair, Y.R., Nabih, Z.T., Philippe, N., Imai, Y., Suzuki, Y., Suh, D.S., Ooi, G.T., 1996. Cloning and characterization of a functional promoter of the rat pp120 gene, encoding a substrate of the insulin receptor tyrosine kinase. J. Biol. Chem. 271, $8809-8817$

Nishi, N., Oya, H., Matsumoto, K., Nakamura, T., Miyanaka, H., Wada, F., 1996. Changes in gene expression of growth factors and their receptors during castration-induced involution and androgeninduced regrowth of rat prostates. Prostate 28, 139-152.

Odin, P., Asplund, M., Busch, C., Obrink, B., 1988. Immunohistochemical localization of CellCAM 105 in rat tissues: appearance in epithelia, platelets, and granulocytes. J. Histochem. Cytochem. 36, 729-739.

Peehl, D.M., Rubin, J.S., 1995. Keratinocyte growth factor: an androgen-regulated mediator of stromal-epithelial interactions in the prostate. World J. Urol. 13, 312-317.

Pu, Y.S., Luo, W., Lu, H.-H., Gingrich, J., Greenberg, N., Lin, S.-H., 1999. Differential expression of C-CAM protein in prostate cancer progression in a transgenic adenocarcinoma mouse model. J. Urol. $162,892-896$.

Roche, P.J., Hoare, S.A., Parker, M.G., 1992. A consensus DNA-binding site for the androgen receptor. Mol. Endocrinol. 6, 2229-2235.

Rubin, J.S., Bottaro, D.P., Chedid, M., Miki, T., Ron, D., Cheon, H.-G., Taylor, W.G., Fortney, E., Sakata, H., Finch, P.W., LaRochelle, W.J., 1995. Keratinocyte growth factor. Cell Biol. Int. 19, 399-411.

Schmitz, M.L., Baeuerle, P.A., 1991. The p65 subunit is responsible for the strong transcription activating potential of NF-kappa B. EMBO J. $10,3805-3817$.

Spencer, T.E., Jenster, G., Burcin, M.M., Allis, C.D., Zhou, J., Mizzen, C.A., McKenna, N.J., Onate, S.A., Tsai, S.Y., Tsai, M.-J., O’Malley, B.W., 1997. Steroid receptor coactivator-1 is a histone acetyltransferase. Nature 389, 194-198.

Sui, X., Bramlett, K.S., Jorge, M.C., Swanson, D.A., von Eschenbach, A.C., Jenster, G., 1999. Specific androgen receptor activation by an artificial coactivator. J. Biol. Chem. 274, 9449-9454.

Yan, G., Fukabori, Y., Nikolaropoulos, S., Wang, F., McKeehan, W.L., 1992. Heparin-binding keratinocyte growth factor is a candidate stromal to epithelial cell andromedin. Mol. Endocrinol. 6, 2123-2128.

Zhang, J., Zhang, S., Murtha, P.E., Zhu, W., Hou, S.S.M., Young, C.Y.F., 1997. Identification of two novel cis-elements in the promoter of the prostate-specific antigen gene that are required to enhance androgen receptor-mediated transactivation. Nucl. Acids Res. 15, 3143-3150.

Zuck, B., Goepfert, C., Nedlin-Chittka, A., Sohrt, K., Voight, K.D., Knabbe, C., 1992. Regulation of fibroblast growth factor-like proteins in the androgen-responsive human prostate cell line, LNCaP. J. Steroid Biochem. Mol. Biol. 41, 659-663. 